\title{
Impact of surgical methodology on the complication rate and functional outcome of patients with a single brain metastasis
}

\author{
*Akash J. Patel, MD, ${ }^{1,2}$ Dima Suki, PhD, ${ }^{1}$ Mustafa Aziz Hatiboglu, MD, ${ }^{1}$ Vikas Y. Rao, MD, ${ }^{2}$ \\ Benjamin D. Fox, MD, ${ }^{2}$ and Raymond Sawaya, MD ${ }^{1,2}$ \\ 1Department of Neurosurgery, The University of Texas M.D. Anderson Cancer Center; and ${ }^{2}$ Department of Neurosurgery, \\ Baylor College of Medicine, Houston, Texas
}

\begin{abstract}
OBJECT Brain metastases are the most common intracranial neoplasms and are on the increase. As radiation side effects are increasingly better understood, more patients are being treated with surgery alone with varying outcomes. The authors previously reported that en bloc resection of a single brain metastasis was associated with decreased incidences of leptomeningeal disease and local recurrence compared with piecemeal resection. However, en bloc resection is often feared to cause an increased incidence of postoperative complications. This study aimed to answer this question. METHODS The authors reviewed data from patients with a previously untreated single brain metastasis, who were treated with resection at The University of Texas M.D. Anderson Cancer Center (1993-2012). Data related to the patient, tumor, and methods of resection were obtained. Discharge Karnofsky Performance Scale (KPS) scores and 30-day postoperative complications were noted. Complications were considered major when they persisted for longer than 30 days, resulted in hospitalization or prolongation of hospital stay, required aggressive treatment, and/or were life threatening.
\end{abstract}

RESULTS During the study period, 1033 eligible patients were identified. The median age was 58 years, $83 \%$ had a KPS score greater than 70 , and $81 \%$ were symptomatic at surgery. Sixty-two percent of the patients underwent en bloc resection of their tumor, and $38 \%$ underwent piecemeal resection. There were significant differences between the 2 groups in terms of preoperative tumor volume, tumor functional grade, and symptoms at presentation, among others. The overall complication rates were $13 \%$ for patients undergoing en bloc resection and $19 \%$ for patients undergoing piecemeal resection $(p=0.007)$. The incidences of major complications and neurological complications were also significantly different. There was a trend in the same direction for major neurological complications, although it was not significant. Among patients undergoing piecemeal resection of tumors in eloquent cortex, $24 \%$ had complications $13 \%$ had major, $18 \%$ had neurological, $9 \%$ had major neurological, and $13 \%$ had select neurological complications; $4 \%$ died within 1 month of surgery). Among those undergoing en bloc resection of such tumors, $11 \%$ had complications (6\% had major, $8 \%$ had neurological, $4 \%$ had major neurological, and $4 \%$ had select neurological; $2 \%$ died within 1 month of surgery). The differences in overall, major, neurological, and select neurological complications were statistically significant, but 1-month mortality and major neurological complications were not. In addition, within subcategories of tumor volume, the incidence of various complications was generally higher for patients undergoing piecemeal resection than for those undergoing en bloc resection.

CONCLUSIONS The authors' results indicate that postoperative complication rates are not increased by en bloc resection, including for lesions in eloquent brain regions or for large tumors. This gives credence to the idea that en bloc resection of brain metastases, when feasible, is at least as safe as piecemeal resection.

http://thejns.org/doi/abs/10.3171/2014.9.JNS13939

KEY WORDS brain metastases; en bloc; craniotomy; complications; functional outcome; oncology

ABBREVIATIONS KPS = Karnofsky Performance Scale; TFG = tumor functional grade .

SUBMITTED May 8, 2013. ACCEPTED September 24, 2014.

INCLUDE WHEN CITING Published online March 20, 2015; DOI: 10.3171/2014.9.JNS13939.

DISCLOSURE The authors report no conflict of interest concerning the materials or methods used in this study or the findings specified in this paper. This work was supported by financial assistance from the Apache Corporation Brain Tumor Data Management Fund.

* Drs. Patel and Suki contributed equally to this work. 
$\mathrm{B}$ RAIN metastases are the most common intracranial neoplasms, with an estimated $10 \%-30 \%$ of cancer patients ultimately developing the condition. . $^{2,31}$ With increased cancer survival, the incidence of brain metastasis has seen a corresponding increase. . $, 7,13,23$ As the side effects of radiation are increasingly better understood, more patients, particularly those with a single brain metastasis, are being treated with surgery alone. However, recurrence rates following this management approach have varied greatly in the published literature, ranging from $10 \%$ to $46 \% .^{9-11,14,22,24}$ Although much attention has been given to identifying various factors that decrease the risk of recurrence, the specific surgical methodology used has not historically been a focus. We previously reported that en bloc resection of a single brain metastasis was associated with the decreased incidence of leptomeningeal disease $^{20,21}$ and local recurrence ${ }^{11}$ compared with piecemeal resection. Our studies have suggested that en bloc resection of metastasis offers several advantages over piecemeal resection. However, en bloc resection is not always achievable. In fact, some surgeons may hesitate to attempt en bloc resection in functionally eloquent areas for fear of increased incidence of postoperative complications, although this has never been specifically studied.

In a large series of neurosurgical patients from our center, tumor functional grade (TFG, a grade that classifies tumors into 3 categories based on proximity to functional cortex: noneloquent [Grade I], near-eloquent [Grade II], and eloquent [Grade III]) was the most important factor affecting the incidence of postoperative neurological deficits. ${ }^{15}$ Previously, we noted a trend for more local recurrence among tumors with higher TFG, but closer examination revealed that more of these tumors were resected in piecemeal fashion ${ }^{11}$ and that surgical method, rather than TFG, was the factor more significantly impacting the incidence of recurrence. To determine if en bloc resection is associated with more complications than piecemeal resection for all tumor locations and particularly those in functionally eloquent brain, we reviewed data from patients with a previously untreated single brain metastasis who were treated with resection from 1993 to 2012 at The University of Texas M.D. Anderson Cancer Center (M.D. Anderson).

\section{Methods}

\section{Patient Population}

The data review was performed under the auspices of an M.D. Anderson institutional review board-approved protocol. Patients were excluded if they had undergone brain tumor surgery, stereotactic radiosurgery, and/or whole-brain radiation therapy prior to the index procedure at M.D. Anderson; if they had a diagnosis of leptomeningeal disease prior to the index procedure; if they were not monitored at M.D. Anderson's Brain and Spine Center after the index procedure; or if they had incomplete data on the entities under study. Using the multidisciplinary Brain and Spine Center Database, we were able to identify 1033 patients with a previously untreated single brain metastasis who underwent surgical resection at our institution between June 1993 and December 2012 and also fit the above criteria.
The following data were reviewed: patient age, sex, Karnofsky Performance Scale (KPS) score (preoperative and at hospital discharge), preoperative tumor volume (including the solid and cystic components of the tumor), TFG, ${ }^{15}$ site of primary cancer, presenting symptoms, type of anesthesia (general, local, or awake), utilization of intraoperative mapping techniques, method of resection (en bloc, defined as circumferential resection of the tumor without violation of the capsule, or piecemeal), extent of tumor resection (based on comparative computerized volumetric analyses of preoperative and postoperative MRI scans), length of postoperative hospital stay, and 30-day postoperative morbidity and mortality. We categorized complications as major or minor. Major complications were those that persisted for more than 30 days, resulted in hospitalization or prolongation of hospital stay, required aggressive treatment, and/or were life threatening. ${ }^{15}$ All other complications were classified as minor. Both new deficits/events and worsening of preoperative deficits/events were counted as complications. Of note, most preoperative and surgical data related to the M.D. Anderson surgeries in this database were obtained prospectively. The method of resection was prospectively noted by the neurosurgeons and entered into the database immediately after the procedure. The radiographic characteristics of the tumor were assessed prospectively. The categorization of complications as major versus minor was done retrospectively, based on a review of medical records. In our series, $96 \%$ of our tumors were completely resected as defined by zero residual tumor or $100 \%$ resection on postoperative MR images, according to computerized analysis.

\section{Statistical Methods}

Frequencies and descriptive statistics on the various entities under study were obtained. Distributions of categorical variables among different subgroups were tested using a chi-square test or the Fisher exact test, as appropriate. Distributions of continuous and ordinal variables were tested using Student's t-test or a nonparametric test, as appropriate. Factors associated with the incidence of 30-day postoperative complications (overall, major, neurological, major neurological, and select neurological complications) were assessed using univariate and multivariate logistic regression analyses. Odds ratios and their $95 \%$ confidence intervals were computed. All tests were 2-tailed. A p value $\leq 0.05$ was considered significant. All analyses were performed using IBM SPSS version 21.

\section{Results}

The patient characteristics are presented in Table 1. Of the 1033 patients, $53 \%$ were males and $47 \%$ were females. The median age of patients at the time of the index procedure was 58 years (range 4-91 years). Eighty-one percent of patients were symptomatic at the time of presentation for surgery, with headache (37\%) being the most common presenting symptom, followed by weakness in $28 \%$, gait difficulty in $20 \%$, visual problems and speech problems in $14 \%$ each, and nausea/vomiting and seizures in 12\% each.

Most of the patients in our study were high functioning at the time of the surgical procedure; i.e., $83 \%$ had a 
TABLE 1. Characteristics of patients who underwent surgery for a single previously untreated brain metastasis $(n=1033)^{*}$

\begin{tabular}{|c|c|c|c|c|}
\hline Characteristic & All Patients $(n=1033)$ & Piecemeal Resection $(n=395)$ & En Bloc Resection $(n=638)$ & $p$ Value \\
\hline Sex & & & & 0.77 \\
\hline Male & $551(53)$ & $213(54)$ & $338(53)$ & \\
\hline Female & $482(47)$ & $182(46)$ & $300(47)$ & \\
\hline Age in yrs & & & & 0.84 \\
\hline Median & 58 & 58 & 58 & \\
\hline Range & $4-91$ & $4-91$ & $4-90$ & \\
\hline Preop KPS score & & & & $<0.001$ \\
\hline Median & 90 & 90 & 90 & \\
\hline Range & $20-100$ & $20-100$ & $40-100$ & \\
\hline Preop KPS score & & & & 0.03 \\
\hline$\leq 70$ & $175(17)$ & $80(20)$ & $95(15)$ & \\
\hline$>70$ & $858(83)$ & $315(80)$ & $543(85)$ & \\
\hline Symptomatic presentation & & & & $<0.001$ \\
\hline No & $195(19)$ & $53(13)$ & $142(22)$ & \\
\hline Yes & $838(81)$ & $342(87)$ & $496(78)$ & \\
\hline \multicolumn{5}{|l|}{ Symptoms } \\
\hline Headache & $378(37)$ & $161(41)$ & $217(34)$ & 0.03 \\
\hline Weakness & $285(28)$ & $125(32)$ & $160(25)$ & 0.02 \\
\hline Gait difficulty & $209(20)$ & $96(24)$ & $113(18)$ & 0.01 \\
\hline Visual deficit & $149(14)$ & $50(13)$ & $99(16)$ & 0.20 \\
\hline Speech difficulty & $144(14)$ & $57(14)$ & $87(14)$ & 0.72 \\
\hline Nausea/vomiting & $129(12)$ & $61(15)$ & $68(11)$ & 0.02 \\
\hline Seizure & $129(12)$ & $45(11)$ & $84(13)$ & 0.40 \\
\hline Sensory change & $101(10)$ & $41(10)$ & $60(9)$ & 0.61 \\
\hline Memory deficit & $98(9)$ & $37(9)$ & $61(10)$ & 0.92 \\
\hline Dizziness & $85(8)$ & $38(10)$ & $47(7)$ & 0.20 \\
\hline Mental status change & $71(7)$ & $27(7)$ & $44(7)$ & 0.97 \\
\hline Confusion & $55(5)$ & $28(7)$ & $27(4)$ & 0.05 \\
\hline Cranial nerve deficit & $34(3)$ & $22(6)$ & $12(2)$ & 0.001 \\
\hline \multicolumn{5}{|l|}{ Primary cancer site } \\
\hline Lung & $325(31)$ & $129(33)$ & $196(31)$ & 0.52 \\
\hline Melanoma & $188(18)$ & $71(18)$ & $117(18)$ & 0.88 \\
\hline Kidney & $124(12)$ & $30(8)$ & $94(15)$ & 0.001 \\
\hline Breast & $125(12)$ & $50(13)$ & $75(12)$ & 0.66 \\
\hline Unknown primary & $25(2)$ & $9(2)$ & $16(2)$ & 0.82 \\
\hline Other & $246(24)$ & $106(27)$ & $140(22)$ & 0.07 \\
\hline \multicolumn{5}{|l|}{ TFG } \\
\hline I (noneloquent) & $332(32)$ & $101(26)$ & $231(36)$ & $<0.001$ \\
\hline II (near-eloquent) & $413(40)$ & $175(44)$ & $238(37)$ & 0.03 \\
\hline III (eloquent) & $288(28)$ & $119(30)$ & $169(26)$ & 0.20 \\
\hline Preop tumor vol in $\mathrm{cm}^{3}$ & & & & $<0.001$ \\
\hline Median & 11.4 & 16.6 & 9.0 & \\
\hline Range & $0.03-141.8$ & $0.2-141.8$ & $0.03-111.2$ & \\
\hline Necrosis & & & & 0.22 \\
\hline No & $587(57)$ & $215(54)$ & $372(58)$ & \\
\hline Yes & $446(43)$ & $180(46)$ & $266(42)$ & \\
\hline Hemorrhagic tumor on imaging & & & & 0.01 \\
\hline No & $846(82)$ & $308(78)$ & $536(84)$ & \\
\hline Yes & $187(18)$ & $87(22)$ & $100(16)$ & \\
\hline
\end{tabular}


TABLE 1. Characteristics of patients who underwent surgery for a single previously untreated brain metastasis $(n=1033)^{*}($ continued)

\begin{tabular}{|c|c|c|c|c|}
\hline Characteristic & All Patients ( $n=1033$ ) & Piecemeal Resection $(n=395)$ & En Bloc Resection $(n=638)$ & $\mathrm{p}$ Value \\
\hline Cystic tumor on imaging & & & & 0.01 \\
\hline No & $898(87)$ & $330(84)$ & $568(89)$ & \\
\hline Yes & $135(13)$ & $65(16)$ & $70(11)$ & \\
\hline Anesthesia & & & & 1.00 \\
\hline General & $1016(98)$ & $389(98)$ & $627(98)$ & \\
\hline Local & $3(0.3)$ & $1(0.3)$ & $2(0.3)$ & \\
\hline Awake & $14(1)$ & $5(1)$ & $9(1)$ & \\
\hline Intraop mapping & & & & 0.89 \\
\hline No & $790(76)$ & $303(77)$ & $487(76)$ & \\
\hline Yes & $243(24)$ & $92(23)$ & $151(24)$ & \\
\hline
\end{tabular}

* Values are number of patients (\%) unless indicated otherwise.

KPS score greater than 70 . The most common primary tumor treated was lung cancer $(31 \%)$, followed by melanoma (18\%), breast cancer (12\%), kidney cancer (12\%), and others (26\%). Tumors were categorized preoperatively according to TFG-332 tumors were Grade I (32\%), 413 were Grade II (40\%), and 288 were Grade III (28\%), 135 tumors $(13 \%)$ had a cystic component, and $187(18 \%)$ were hemorrhagic. The median preoperative tumor volume was $11.4 \mathrm{~cm}^{3}$ (range $0.03-141.8 \mathrm{~cm}^{3}$ ). Most of the patients were administered general anesthesia (98\%), with $3(0.3 \%)$ being administered local anesthesia, and 14 (1\%) undergoing awake craniotomy (this variable was not reviewed further). Intraoperative mapping was used in 243 (24\%) of the cases.

Sixty-two percent of the patients underwent en bloc resection of the tumor, and $38 \%$ underwent piecemeal resection. We compared all characteristics between patients undergoing en bloc resection and those undergoing piecemeal resection (Table 1). The median preoperative tumor volume was $16.6 \mathrm{~cm}^{3}$ for those undergoing piecemeal resection and $9.0 \mathrm{~cm}^{3}$ for those undergoing en bloc resection, a statistically significant difference $(\mathrm{p}<0.001)$. TFG was unequally distributed between the 2 surgical methods, with a significantly larger proportion of TFG II in the piecemeal group $(p=0.03)$ and a significantly larger proportion of TFG I in the en bloc group (p < 0.001). A significantly larger proportion of patients undergoing piecemeal resection rather than en bloc resection were symptomatic at presentation $(87 \%$ vs $78 \%$, respectively; $\mathrm{p}<0.001)$. A significantly larger proportion of patients undergoing piecemeal resection had cystic tumors $(16 \%$ vs $11 \%$, respectively; $p=0.01$ ), and hemorrhagic tumors ( $22 \%$ vs $16 \%$, respectively; $p=0.01$ ). In addition, the en bloc resection group contained significantly larger proportions of patients with a renal cell primary cancer $(\mathrm{p}=$ $0.001)$ and patients with a KPS score greater than $70(\mathrm{p}=$ 0.03 ). There were no other statistically significant differences in characteristics between the piecemeal and en bloc groups, including with respect to TFG III tumors.

Postoperative hospital length of stay was significantly longer among patients in the piecemeal group (median, 4.0 days; range 2-47 days) than for those in the en bloc group (median, 3.0 days; range $1-78$ days; $p<0.001$; Table 2).
The KPS score at hospital discharge remained within 10 points of the preoperative score in 924 patients (89\%), worsened by greater than 10 points in 57 patients $(6 \%)$, and improved by greater than 10 in 52 patients (5\%). Among patients undergoing piecemeal resection, $338(86 \%)$ had a discharge KPS score within 10 points of their preoperative score, compared with 586 patients $(92 \%)$ in the en bloc group $(\mathrm{p}=0.001)$. There was no significant difference in the proportion of patients whose KPS score worsened after surgery ( $6 \%$ vs $5 \%$ in the piecemeal and en bloc groups, respectively; $p=0.40$ ), but more patients improved in the piecemeal group than in the en bloc group (8\% and $3 \%$, respectively; $\mathrm{p}<0.001$ ).

Thirty-day mortality was 3\%, with 34 of 1033 patients dying within 1 month of surgery (18 [5\%] in the piecemeal group and 16 [2\%] in the en bloc group, $\mathrm{p}=0.07$ ). Table 3 shows the incidence of overall complications within 1 month after surgery in our cohort, as well as the incidence of major, neurological, and major neurological complications during that period. The overall complication rates were $13 \%$ for patients undergoing en bloc resection and $19 \%$ for patients undergoing piecemeal resection $(\mathrm{p}=$ $0.007)$. There was a significant difference in the incidence of major complications between the 2 groups ( $7 \%$ vs $10 \%$, respectively; $p=0.04$ ). These differences remained significant in the multivariate logistic regression analysis after controlling for other factors associated with complications and major complications (OR 0.62 [95\% CI 0.43-0.88], $\mathrm{p}=0.008$ and OR 0.61 [95\% CI] 0.39-0.96], $\mathrm{p}=0.03$; respectively). These differences also remained significant in the multivariate logistic regression analysis after controlling for other factors associated with neurological and select neurological complications (OR 0.55 [95\% CI $0.36-0.84], \mathrm{p}=0.006$ and OR 0.46 [95\% CI, 0.27-0.81], $\mathrm{p}=0.007$; respectively). There was a trend in the same direction for major neurological complications, though it was not significant (OR 0.63 [95\% CI 0.35-1.13], $\mathrm{p}=0.12$ ).

In addition to surgical method, several other factors significantly impacted the incidence of overall complications in the multivariate analysis: older patients had a significantly higher rate of overall complications (OR 1.02 [95\% CI 1.01-1.04], $\mathrm{p}=0.002$ ), as did patients with a renal primary tumor (OR 1.92 [95\% CI 1.18-3.11], $\mathrm{p}=0.008)$. In 
TABLE 2. Postoperative treatment outcomes in patients who underwent surgery for a single previously untreated brain metastasis*

\begin{tabular}{|c|c|c|c|c|}
\hline Outcome & All Patients $(n=1033)$ & Piecemeal Resection $(n=395)$ & En Bloc Resection $(n=638)$ & $\mathrm{p}$ Value \\
\hline Postop LOS in mos & & & & $<0.001$ \\
\hline Median & 3 & 4 & 3 & \\
\hline Range & $1-78$ & $2-47$ & $1-78$ & \\
\hline KPS score comparison† & & & & 0.001 \\
\hline Worse by $>10$ & $57(6)$ & $25(6)$ & $32(5)$ & \\
\hline Same & $924(89)$ & $338(86)$ & $586(92)$ & \\
\hline Improved by $>10$ & $52(5)$ & $32(8)$ & $20(3)$ & \\
\hline KPS score comparison† & & & & 0.001 \\
\hline Worse by >40 & $15(1)$ & $7(2)$ & $8(1)$ & \\
\hline Worse by 40 & $3(0.3)$ & $3(0.8)$ & $0(0)$ & \\
\hline Worse by 30 & $10(1)$ & $4(1)$ & $6(0.9)$ & \\
\hline Worse by 20 & $29(3)$ & $11(3)$ & $18(3)$ & \\
\hline Same & $924(89)$ & $338(86)$ & $586(92)$ & \\
\hline Improved by 20 & $34(3)$ & $21(5)$ & $13(2)$ & \\
\hline Improved by 30 & $9(0.9)$ & $3(0.8)$ & $6(0.9)$ & \\
\hline Improved by 40 & $6(0.6)$ & $5(1)$ & $1(0.2)$ & \\
\hline Improved by $>40$ & $3(0.3)$ & $3(0.8)$ & $0(0)$ & \\
\hline 1-mo mortality & & & & 0.07 \\
\hline Alive & 999 (97) & 377 (95) & $622(98)$ & \\
\hline Dead & $34(3)$ & $18(5)$ & $16(2)$ & \\
\hline
\end{tabular}

LOS = length of stay.

* Values are number of patients (\%) unless stated otherwise.

$\dagger$ Discharge KPS score - preoperative KPS score.

addition, the use of mapping was associated with a higher rate of overall complications (OR 1.55 [95\% CI 1.05$2.29], \mathrm{p}=0.03$ ). Patients with a higher KPS score, on the other hand, had a lower incidence of complications (OR 0.98 [95\% CI 0.97-0.99], $\mathrm{p}=0.007$ ). Of note, the overall complication rates according to TFG were $12 \%, 16 \%$, and $17 \%$ for Grades I, II, and III, respectively (overall $\mathrm{p}=$ 0.27 ), with respective univariate ORs of 1.33 and 1.42 for Grades II and III compared with Grade I, respectively ( $\mathrm{p}$ $=0.19$ and 0.13 ).

Similarly, several other factors significantly impacted the incidence of overall complications in the multivariate analysis: older patients had a significantly higher rate of overall complications (OR 1.03 [95\% CI 1.01-1.04], $\mathrm{p}=$ $0.006)$. In addition, the use of mapping was associated with a higher complication rate (OR 1.74 [95\% CI 1.07-2.81]; $\mathrm{p}$ $=0.02$ ). The major complication rates by TFG were $6 \%$, 9\%, and 9\% for Grades I, II, and III, respectively (overall $p=0.15$ ), with respective univariate ORs of 1.67 and 1.70 for Grades II and III compared with Grade I, respectively $(\mathrm{p}=0.08$ and 0.09). Similar factors impacted neurological complications. Of note, the presence of preoperative symptoms was significantly associated with the incidence of neurological complications, and TFG was significantly associated with the incidence of select neurological complications in the multivariate analysis (data not shown).

We also assessed the 30-day neurological complication rate (Tables 3 and 4). One hundred four patients (10\%) had 1 or more neurological deficit(s). These included focal motor deficits (4\%), speech impairment (3\%), and seizures (1\%). Hydrocephalus, visual impairment, focal sensory deficit, bladder dysfunction, cranial nerve deficits, and cerebral abscess were each observed in less than $1 \%$ of instances. Intracranial hemorrhage occurred in 14 patients $(1 \%$; 8 intraparenchymal, 4 epidural, and 2 subdural). Patients undergoing piecemeal resection had a higher incidence of neurological complications than those undergoing en bloc resection (13\% vs $8 \%$, respectively; $\mathrm{p}=0.03$ ), although the incidence of major neurological complications was not significantly different between the 2 groups (6\% vs $4 \%$ in the piecemeal and en bloc groups, respectively; $\mathrm{p}=0.18$ ). The piecemeal group had a higher incidence of speech impairment than the group undergoing en bloc resection $(\mathrm{p}=0.001)$.

We grouped together motor, sensory, speech, and visual deficits as "select" neurological complications. The rate of select neurological complications across all patients was $8 \%$ in the piecemeal group and 5\% in the en bloc group; $\mathrm{p}=0.04)$.

A tumor's functional grade is often a major deciding factor in the selection of method of resection and could impact the incidence of complications, particularly neurological complications, by method. We therefore focused our secondary analysis on patients with tumors in eloquent brain (TFG III). Of note, among patients with TFG I and II lesions, the rates of complications (overall, major, neurological, major neurological, select neurological, and 1-month mortality) were not significantly different between patients undergoing piecemeal and en bloc resection. Among patients undergoing resection of tumors in eloquent cortex, 


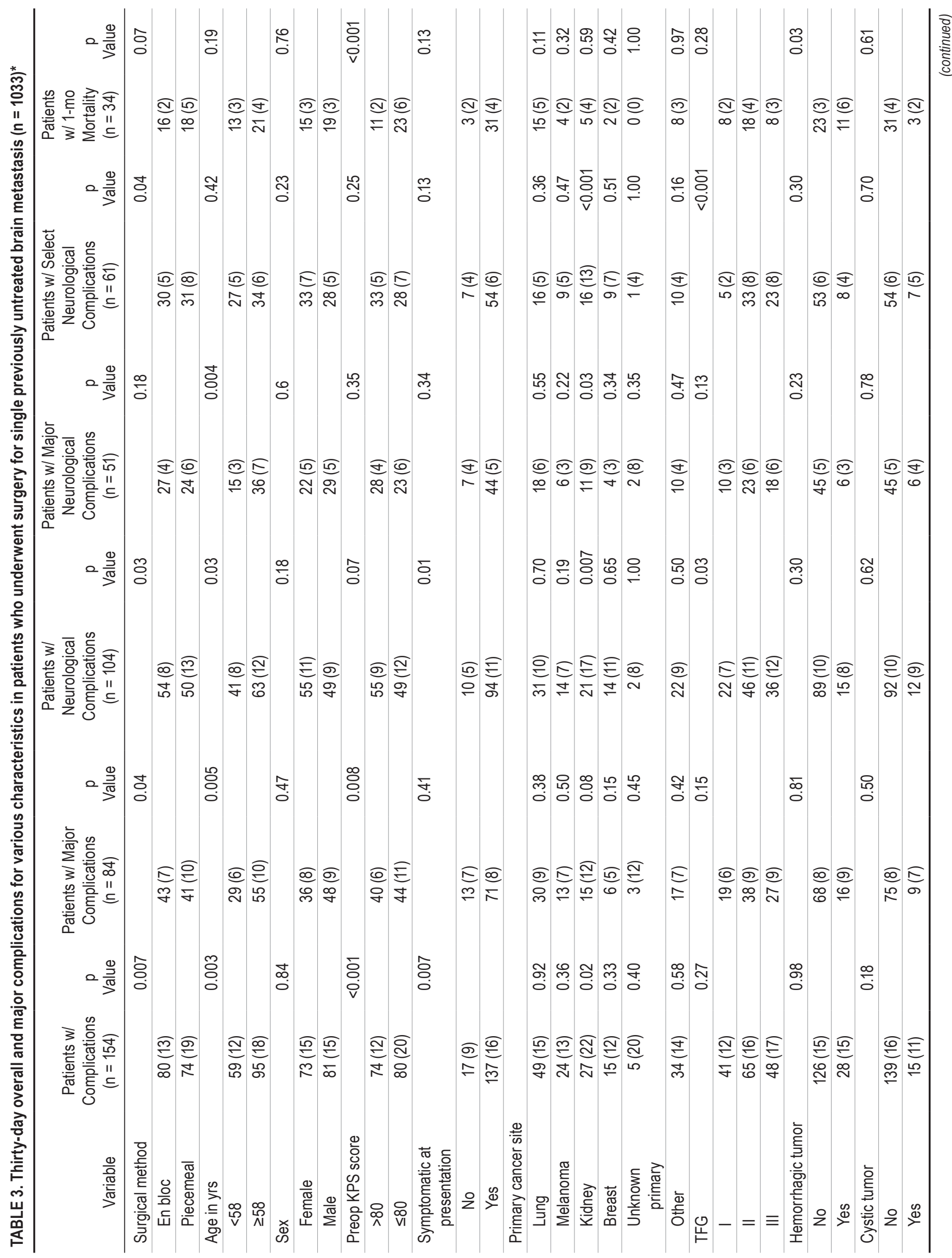




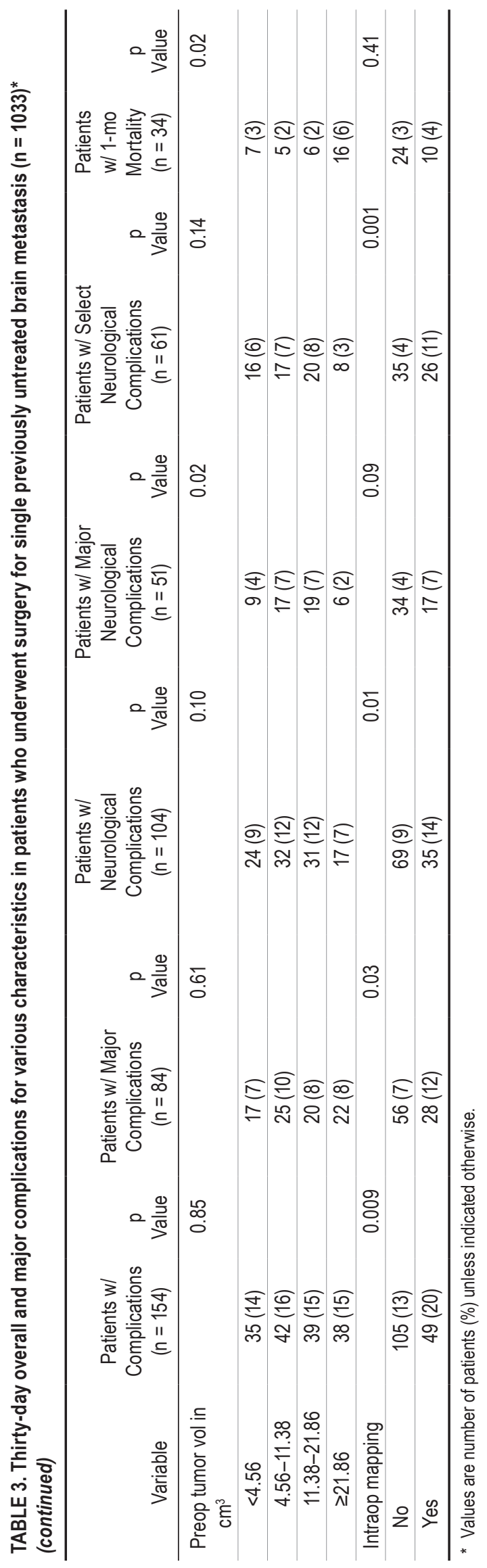

48 patients (17\%) had complications, 27 (9\%) had major complications, 36 (12\%) had neurological complications, and $23(8 \%)$ had select neurological complications. Among patients undergoing piecemeal resection of tumors in eloquent cortex, 29 patients (24\%) had complications, $16(13 \%)$ had major complications, 22 (18\%) had neurological complications, 11 (9\%) had major neurological complications, 16 (13\%) had select neurological complications, and 5 (4\%) patients died within 1 month of surgery. Among those undergoing en bloc resection of such tumors, 19 (11\%) had complications, $11(6 \%)$ had major complications, $14(8 \%)$ had neurological complications, 7 (4\%) had major neurological complications, 7 (4\%) had select neurological complications, and $3(2 \%)$ patients died within 1 month of surgery (Fig. 1). The differences in patients undergoing piecemeal versus en bloc resection with respect to overall, major, neurological, and select neurological complications were statistically significant, but not with respect to 1-month mortality and major neurological complications.

Among patients with a tumor in eloquent brain, the discharge KPS score remained within 10 points of the preoperative score in 257 patients (89\%), worsened by greater than 10 points in 15 patients $(5 \%)$, and improved by greater than 10 points in 16 patients $(6 \%)$. Ninety-nine patients $(83 \%)$ who underwent piecemeal resection had a KPS score within only 10 points of the preoperative score at discharge, relative to 158 patients $(94 \%)$ in the en bloc group $(\mathrm{p}=0.005)$. Nine patients $(8 \%)$ who underwent piecemeal resection had a worsening in KPS score of more than 10 points versus 6 patients $(4 \%)$ who underwent en bloc resection ( $\mathrm{p}=0.13) ; 11$ patients $(9 \%)$ who underwent piecemeal resection had an improvement in KPS by more than 10 points versus 5 patients $(3 \%)$ who underwent en bloc resection $(\mathrm{p}=0.02)$.

Preoperative tumor volume is another major deciding factor in the selection of method of resection. We closely reviewed our data to determine if there was a differential effect of method of resection on incidence of 30-day postoperative complications relative to preoperative tumor volume. The analysis showed that within subcategories of tumor volume, the incidence of various complications is generally higher for patients undergoing piecemeal resection than for those undergoing en bloc resection (Fig. 2).

\section{Discussion}

Previously, we found that en bloc resection was associated with a lower incidence of leptomeningeal disease ${ }^{20,21}$ and local recurrence ${ }^{11}$ than piecemeal resection. One important unanswered question is whether performing an en bloc resection in patients with brain metastasis, and in particular those with tumors in functionally eloquent brain, results in an increased risk of postoperative deficits. We reviewed data from patients with a previously untreated single brain metastasis that was treated with resection at M.D. Anderson.

Our primary question in embarking on this study was to determine whether method of resection, en bloc versus piecemeal, impacted the patients' functional outcomes and complication rates postoperatively. In our cohort, more patients underwent en bloc resection (638 patients; 62\%) 
TABLE 4. Overall 30-day complications and complications according to surgical method in patients who underwent surgery for a single previously untreated brain metastasis*

\begin{tabular}{|c|c|c|c|c|}
\hline Complication & All Patients $(n=1033)$ & Piecemeal Resection $(n=395)$ & En Bloc Resection $(n=638)$ & $\mathrm{p}$ Value \\
\hline Overall complications & $154(15)$ & $74(19)$ & $80(13)$ & 0.007 \\
\hline Major complications & $84(8)$ & $41(10)$ & $43(7)$ & 0.04 \\
\hline All neurological complications & $104(10)$ & $50(13)$ & $54(8)$ & 0.03 \\
\hline Focal motor deficit & $44(4)$ & $21(5)$ & $23(4)$ & 0.19 \\
\hline Focal sensory deficit & $5(0.5)$ & $2(0.5)$ & $3(0.5)$ & 1.00 \\
\hline Speech impairment & $28(3)$ & $19(5)$ & $9(1)$ & 0.001 \\
\hline Visual impairment & $6(0.6)$ & $2(0.5)$ & $4(0.6)$ & 1.00 \\
\hline Cranial nerve deficit & $2(0.2)$ & $1(0.3)$ & $1(0.2)$ & 1.00 \\
\hline Bladder dysfunction & $4(0.4)$ & $3(0.8)$ & $1(0.2)$ & 0.16 \\
\hline Hydrocephalus & $9(0.9)$ & $5(1)$ & $4(0.6)$ & 0.32 \\
\hline Seizure & $13(1)$ & $4(1)$ & $9(1)$ & 0.78 \\
\hline Major neurological complications & $51(5)$ & $24(6)$ & $27(4)$ & 0.18 \\
\hline Select neurological complications $†$ & $61(6)$ & $31(8)$ & $30(5)$ & 0.04 \\
\hline Intracranial hemorrhage & $14(1)$ & $3(0.8)$ & $11(2)$ & 0.19 \\
\hline Subdural & $2(0.2)$ & $1(0.3)$ & $1(0.2)$ & 1.00 \\
\hline Epidural & $4(0.4)$ & $1(0.3)$ & $3(0.5)$ & 1.00 \\
\hline Intraparenchymal & $8(0.8)$ & $1(0.3)$ & $7(1)$ & 0.16 \\
\hline \multicolumn{5}{|l|}{ Infection } \\
\hline Meningitis & $3(0.3)$ & $2(0.5)$ & $1(0.2)$ & 0.56 \\
\hline Wound infection & $7(0.7)$ & $0(0.0)$ & $7(1)$ & 0.05 \\
\hline \multicolumn{5}{|l|}{ Peripheral/vascular } \\
\hline Pulmonary embolus & $2(0.2)$ & $1(0.3)$ & $1(0.2)$ & 1.00 \\
\hline Deep venous thrombosis & $11(1)$ & $5(1)$ & $6(0.9)$ & 0.76 \\
\hline
\end{tabular}

* Values in parentheses are percentages.

$\dagger$ Motor, sensory, speech, and visual deficits.

than piecemeal resection (395 patients; 38\%). Patients undergoing piecemeal resection had an overall complication rate of $19 \%$ and a major complication rate of $10 \%$. Patients undergoing en bloc resection had an overall complication rate of $13 \%$ and a major complication rate of $7 \%(\mathrm{p}=0.007$ and 0.04 , respectively, for the 2 comparisons). In fact, in the univariate analysis, we found that piecemeal resection had a higher rate of overall, major, neurological, and select neurological complications, as well as speech impairment, than en bloc resection. These differences were all statistically significant. Therefore, the response to the first question posed by this paper is that en bloc resection is not associated with an increased risk of most complications compared with piecemeal resection, but the opposite is actually true.

There were some notable statistically significant differences between the piecemeal and en bloc groups in our cohort. The former group had significantly larger tumor volumes, more tumors near or within eloquent brain, and more patients with low KPS scores. Patients undergoing piecemeal resection also were more frequently symptomatic at presentation (87\% vs $78 \%$, among those undergoing en bloc resection; $\mathrm{p}<0.001$ ). This difference is probably related to the larger sizes of tumors in the piecemeal group, to their more frequent location within or near eloquent brain, or both. However, a closer look within the various subcategories of tumor volume or tumor functional grade shows that en bloc resection did not result in a higher frequency of complications (overall and other).

It seems intuitive that tumor location in relation to eloquent brain is a critical factor in determining the risk of postoperative complications. Our group previously reported the importance of tumor functional grade in the incidence of postoperative neurological deficits in a group of patients harboring tumors with mixed histologies..$^{15}$ The issue of tumor location was deemed less relevant because a tumor located within or near eloquent brain is accessed through noneloquent brain tracts regardless of whether it is deeply or superficially located. This led to the second question posed in this paper: Do the above findings of a higher incidence of complications in patients undergoing piecemeal versus en bloc resection of their tumor hold true for tumors located within eloquent brain? As a result, tumors were classified according to proximity to eloquent brain, and a separate analysis was done for TFG III tumors. These tumors had an overall neurological complication rate of $12 \%$, whereas Grade I tumors had a rate of $7 \%$ $(\mathrm{p}=0.01)$. TFG III tumors also had a significantly higher rate of major neurological complications $(6 \%$ vs $3 \%$, respectively; $p=0.053$ ) and a significantly higher rate of select neurological complications ( $8 \%$ vs $2 \%$, respectively; < $0.001)$, including speech impairment alone. There was only 

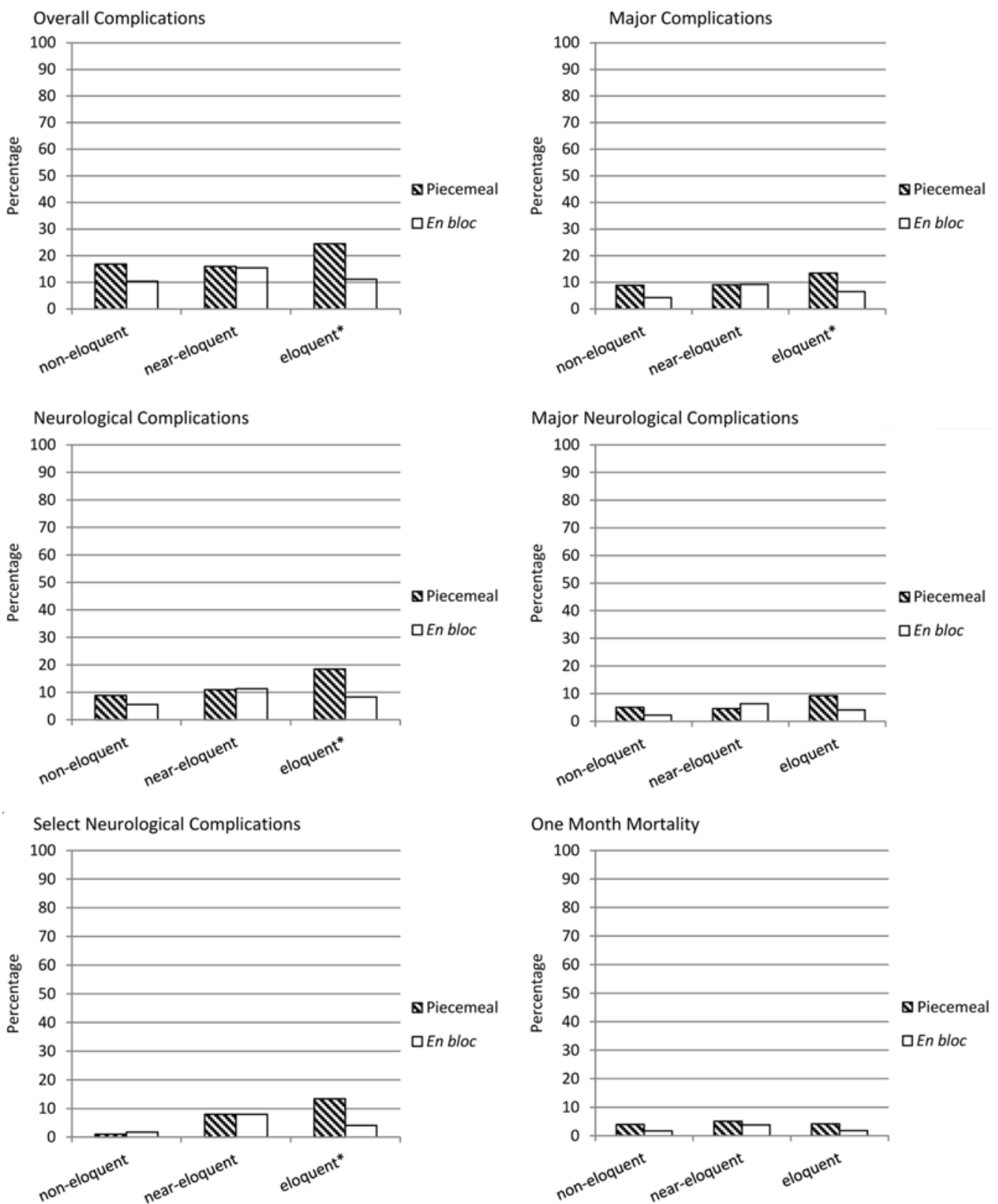

FIG. 1. Incidence of various complications according to preoperative tumor functional grade. ${ }^{*} p \leq 0.05$.

a trend, not a significant one, toward higher complications and major complications ( $\mathrm{p}=0.13$ and 0.08 , respectively).

It also seems intuitive that tumor volume would be a critical factor in determining the risk of postoperative complications. This led to the third question posed in this paper: Do the above findings of a higher incidence of complications in patients undergoing piecemeal versus en bloc resection of their tumor hold true regardless of tumor volume? To investigate this issue, we compared the incidence of complications within narrow categories of preoperative tumor volume. The overall trends in complications according to surgical method remained largely unaltered.

In addition to surgical method, characteristics that we found to significantly affect the overall complication rate in the multivariate analysis were older age, lower KPS score, a requirement for intraoperative mapping, and a kidney primary tumor. Those associated with major complications were older age and a requirement for intraoperative mapping.

Older age has long been suggested as a risk factor for postoperative complications in multiple surgical specialties, $5,12,16,18,19$ although other studies have found the opposite to be true as well. ${ }^{4,8}$ In our study, we found that age was significantly associated with the incidence of both overall and major complications. Although we do not feel that age alone should be a contraindication to surgery, it is important to keep in mind that older age could contribute to increased postoperative morbidity. 

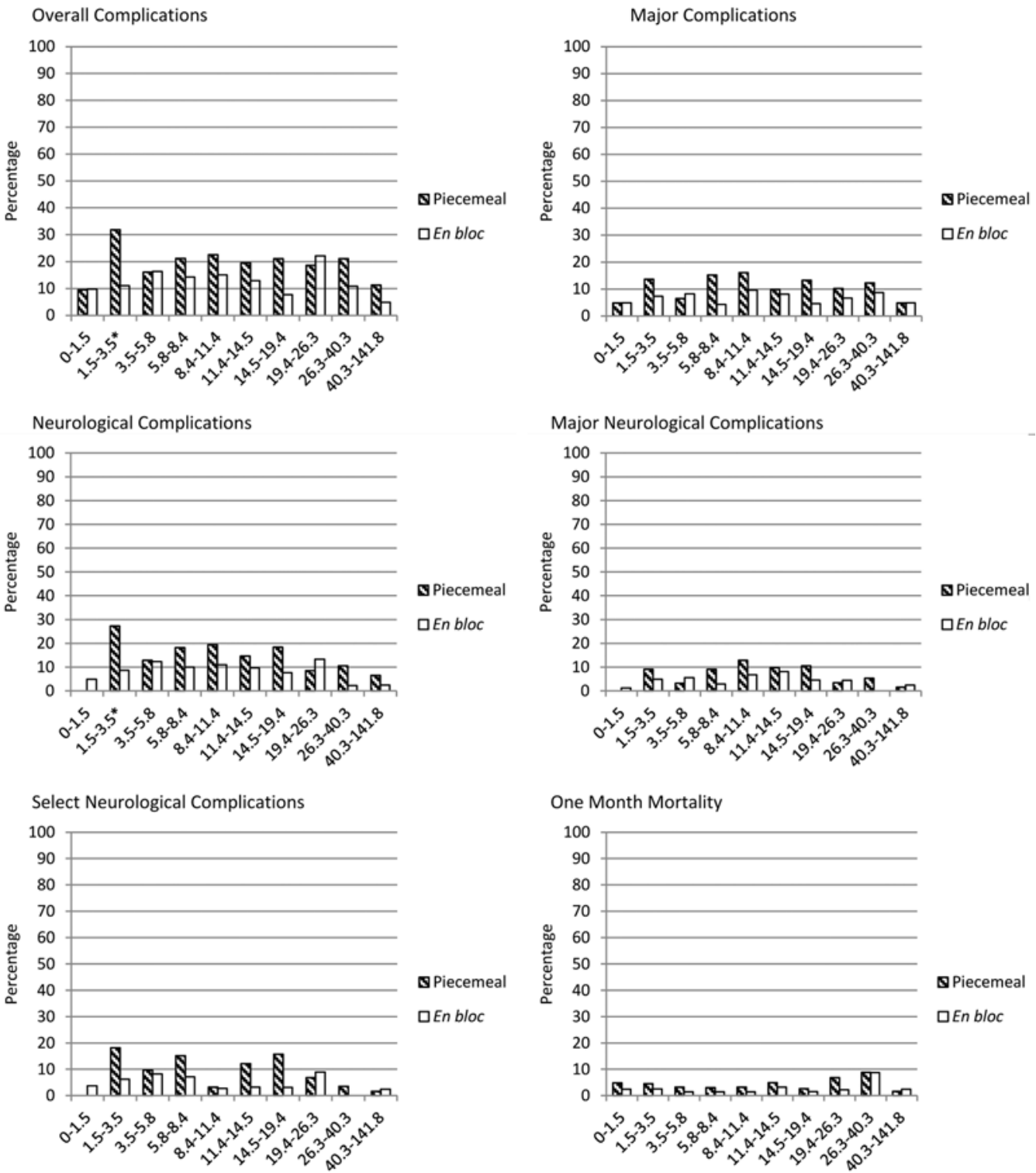

FIG. 2. Incidence of various complications according to preoperative tumor volume category (in $\left.\mathrm{cm}^{3}\right) .{ }^{*} p \leq 0.05$.

As we found in a previous series, ${ }^{15}$ a lower KPS score was associated with a higher rate of complications. The KPS score has always been shown to be a critical factor in various outcomes of treatment, including both complications and survival. ${ }^{1,6,15,17}$ Very low KPS scores are often a contraindication for surgery.

The requirement for cortical mapping is intimately related to TFG. Lesions in eloquent brain underwent intraoperative mapping significantly more often than those in noneloquent or near-eloquent brain.

In our series, patients with a renal cell cancer primary had a higher rate of complications than those with any other primary tumor. Whereas renal cell cancer metastases are known to be highly vascular, this higher rate of surgical complications has not been previously reported. Interestingly, patients with renal cell cancer metastases underwent en bloc resection more frequently than piece- meal resection. This is probably because, at our institution, we feel that en bloc resection of renal cell cancer metastases has the added advantage of limiting blood loss for this pathology, as entering the lesion can be very bloody. These lesions pose a particular dilemma because they are considered relatively radioresistant.

One might expect to encounter new deficits in asymptomatic patients, as they do not have any deficits to mask new complications. In our study, preoperative symptoms were a finding in the analysis of neurological complications. Our definition of complications was the occurrence of a new event or the worsening of preexisting symptoms. Presumably, patients with deficits are more susceptible to worsening of their symptoms after an intervention, given that something is already wrong. Symptoms are also more frequent in patients with tumors located within or near eloquent brain, and these have a higher rate of neurologi- 
cal complications. Future studies may or may not support this finding.

This study has a number of limitations, the main one being that the 2 cohorts are inherently and often different. There were significant differences between the 2 groups in baseline characteristics, such as symptomatic status at presentation for surgery, preoperative KPS score, and primary cancer type, as well as tumor volume and functional grade and the finding of a cystic or hemorrhagic tumor. A closer examination showed that the findings remained basically unaltered when the 2 methods of resection were compared within subgroups of tumor volume, TFG, and other variables. In addition to the known differences between the piecemeal and en bloc groups, there are likely to be other inherent differences in lesions that can be removed in an en bloc versus a piecemeal fashion that are not necessarily easily controlled for with such a study design. In addition, although most of the data were collected in a prospective fashion, the study is a retrospective one. As a result, important information was not available for review, such as the specific reason that the neurosurgeon chose to use one method of resection over another. En bloc resection may not always be possible and may be less likely to be carried out in eloquent brain areas, although this was not exactly the case in our patient population. Data on the 30-day postoperative KPS scores were also incomplete. The KPS scores presented in the study were assessed at hospital discharge. Some of the complications may have taken place after hospital discharge, which explains the seemingly inconsistent finding of a significant improvement in the KPS score in the piecemeal group relative to the en bloc group. Finally, despite the large sample size achieved, the power of the study to detect such rare complications as speech impairment may be limited. Yet, despite these limitations, most of the data (including the postoperative length of stay) pointed in the same direction, which imparts extra confidence to the legitimacy of the conclusion derived from this study, namely that (when possible) en bloc resection does not appear to result in worse outcomes.

\section{Conclusions}

We performed detailed analyses to account for differences in preoperative KPS score, patient age, tumor type, tumor volume, TFG, symptomatic presentation, and cortical mapping, among others, on the differential incidence of various complication categories according to surgical methodology (piecemeal vs en bloc resection). Even after adjusting for these variables, the complication rates were almost always higher among patients treated with a piecemeal resection. The same applied to patients with tumors located in eloquent brain and tumors along the volume spectrum.

Our results indicate that postoperative complication rates are not increased by en bloc resection, including for lesions in eloquent brain regions or for large tumors. This gives credence to the idea that en bloc resection of brain metastasis is at least as safe as piecemeal resection. In conjunction with our previous findings ${ }^{11,20,21}$ that en bloc resection results in lower rates of leptomeningeal disease and local recurrence in somewhat smaller lesions $(<9.71$ $\mathrm{cm}^{3}$ ), we feel that en bloc resection of metastatic lesions, when achievable, is as safe, if not superior to, piecemeal resection. These findings should be confirmed in a larger prospective study.

\section{Acknowledgments}

We thank Miao Li for assistance with imaging analyses and Stephanie Jenkins and David M. Wildrick, Ph.D., for assistance with manuscript preparation.

\section{References}

1. Antoni D, Clavier JB, Pop M, Schumacher C, Lefebvre F, Noël G: Institutional, retrospective analysis of 777 patients with brain metastases: treatment outcomes and diagnosisspecific prognostic factors. Int J Radiat Oncol Biol Phys 86:630-637, 2013

2. Barker FG II: Surgical and radiosurgical management of brain metastases. Surg Clin North Am 85:329-345, 2005

3. Barnholtz-Sloan JS, Sloan AE, Davis FG, Vigneau FD, Lai P, Sawaya RE: Incidence proportions of brain metastases in patients diagnosed (1973 to 2001) in the Metropolitan Detroit Cancer Surveillance System. J Clin Oncol 22:2865-2872, 2004

4. Boruk M, Chernobilsky B, Rosenfeld RM, Har-El G: Age as a prognostic factor for complications of major head and neck surgery. Arch Otolaryngol Head Neck Surg 131:605-609, 2005

5. Buitelaar DR, Balm AJ, Antonini N, van Tinteren H, Huitink JM: Cardiovascular and respiratory complications after major head and neck surgery. Head Neck 28:595-602, 2006

6. Gaspar L, Scott C, Rotman M, Asbell S, Phillips T, Wasserman T, et al: Recursive partitioning analysis (RPA) of prognostic factors in three Radiation Therapy Oncology Group (RTOG) brain metastases trials. Int J Radiat Oncol Biol Phys 37:745-751, 1997

7. Giordana MT, Cordera S, Boghi A: Cerebral metastases as first symptom of cancer: a clinico-pathologic study. J Neurooncol 50:265-273, 2000

8. Matin SF, Abreu S, Ramani A, Steinberg AP, Desai M, Strzempkowski B, et al: Evaluation of age and comorbidity as risk factors after laparoscopic urological surgery. J Urol 170:1115-1120, 2003

9. Nieder C, Astner ST, Grosu AL, Andratschke NH, Molls M: The role of postoperative radiotherapy after resection of a single brain metastasis. Combined analysis of 643 patients. Strahlenther Onkol 183:576-580, 2007

10. Patchell RA, Tibbs PA, Regine WF, Dempsey RJ, Mohiuddin M, Kryscio RJ, et al: Postoperative radiotherapy in the treatment of single metastases to the brain: a randomized trial. JAMA 280:1485-1489, 1998

11. Patel AJ, Suki D, Hatiboglu MA, Abouassi H, Shi W, Wildrick DM, et al: Factors influencing the risk of local recurrence after resection of a single brain metastasis. J Neurosurg 113:181-189, 2010

12. Pessaux P, Msika S, Atalla D, Hay JM, Flamant Y: Risk factors for postoperative infectious complications in noncolorectal abdominal surgery: a multivariate analysis based on a prospective multicenter study of 4718 patients. Arch Surg 138:314-324, 2003

13. Posner JB, Chernik NL: Intracranial metastases from systemic cancer. Adv Neurol 19:579-592, 1978

14. Salvati M, Cervoni L, Delfini R: Solitary brain metastases from non-oat cell lung cancer: clinical and prognostic features. Neurosurg Rev 19:221-225, 1996

15. Sawaya R, Hammoud M, Schoppa D, Hess KR, Wu SZ, Shi WM, et al: Neurosurgical outcomes in a modern series of 
400 craniotomies for treatment of parenchymal tumors. Neurosurgery 42:1044-1056, 1998

16. Schoenfeld AJ, Ochoa LM, Bader JO, Belmont PJ Jr: Risk factors for immediate postoperative complications and mortality following spine surgery: a study of 3475 patients from the National Surgical Quality Improvement Program. J Bone Joint Surg Am 93:1577-1582, 2011

17. Sengöz M, Kabalay IA, Tezcanlı E, Peker S, Pamir N: Treatment of brainstem metastases with gamma-knife radiosurgery. J Neurooncol 113:33-38, 2013

18. Singh B, Cordeiro PG, Santamaria E, Shaha AR, Pfister DG, Shah JP: Factors associated with complications in microvascular reconstruction of head and neck defects. Plast Reconstr Surg 103:403-411, 1999

19. Sukharamwala P, Thoens J, Szuchmacher M, Smith J, DeVito $\mathrm{P}$ : Advanced age is a risk factor for post-operative complications and mortality after a pancreaticoduodenectomy: a metaanalysis and systematic review. HPB (Oxford) 14:649-657, 2012 [Erratum in HPB (Oxford) 14:877, 2012]

20. Suki D, Abouassi H, Patel AJ, Sawaya R, Weinberg JS, Groves MD: Comparative risk of leptomeningeal disease after resection or stereotactic radiosurgery for solid tumor metastasis to the posterior fossa. J Neurosurg 108:248-257, 2008

21. Suki D, Hatiboglu MA, Patel AJ, Weinberg JS, Groves MD, Mahajan A, et al: Comparative risk of leptomeningeal dissemination of cancer after surgery or stereotactic radiosurgery for a single supratentorial solid tumor metastasis. Neurosurgery 64:664-676, 2009
22. Sundaresan N, Galicich JH: Surgical treatment of brain metastases. Clinical and computerized tomography evaluation of the results of treatment. Cancer 55:1382-1388, 1985

23. Vecht CJ: Clinical management of brain metastasis. J Neurol 245:127-131, 1998

24. Yoo H, Kim YZ, Nam BH, Shin SH, Yang HS, Lee JS, et al: Reduced local recurrence of a single brain metastasis through microscopic total resection. J Neurosurg 110:730-736, 2009

\section{Author Contributions}

Conception and design: Sawaya, Patel, Suki. Acquisition of data: Patel, Suki, Hatiboglu, Rao, Fox. Analysis and interpretation of data: all authors. Drafting the article: Sawaya, Patel, Suki. Critically revising the article: all authors. Reviewed submitted version of manuscript: Sawaya, Patel, Hatiboglu, Rao, Fox. Approved the final version of the manuscript on behalf of all authors: Sawaya. Statistical analysis: Patel, Suki. Study supervision: Sawaya.

\section{Correspondence}

Raymond Sawaya, Department of Neurosurgery, The University of Texas M.D. Anderson Cancer Center, 1515 Holcombe Blvd., Unit 442, Houston, TX 77030-4009. email: rsawaya@ mdanderson.org. 